\title{
Fourier Transforms \\ By \\ White-Light Interferometry: Michelson Stellar Interferometer Fringes
}

\author{
James B. Breckinridge \\ California Institute of Technology, Pasadena, CA \\ And \\ College of Optical Sciences \\ University of Arizona, Tucson, AZ
}

\begin{abstract}
The white-light compensated rotational shear interferometer (coherence interferometer) was developed in an effort to study the spatial frequency content of passively illuminated white-light scenes in real-time and to image sources of astronomical interest at high spatial frequencies through atmospheric turbulence. This work was inspired by Professor Goodman's studies of the image formation properties of coherent (laser) illuminated transparencies. We discovered that real-time image processing is possible using white-light interferometry. The concept of a quasimonoplanatic approximation is introduced as a parallel to the quasimonochromatic approximation needed to describe the theory of Fourier transform spectrometers. This paper describes the coherence interferometer and reviews its image formation properties under the conditions of quasimonoplanacity and describes its development and its applications to physical optics, optical processing and astrophysics including the search for exoplanets.
\end{abstract}

Keywords: rotational shear interferometry, astronomy, atmospheric optics, image processing, coherence theory \& measurement, Michelson stellar interferometry, quasimonoplanatic

\section{INTRODUCTION}

Goodman $^{1}$ in 1968 showed that the two-dimensional spatial frequency Fourier transform of a scene can be recorded when the scene is a transparency positioned at the pupil of an imaging system and illuminated with laser light. The Fourier transform appears encoded in intensity across the focal plane of the optical system. The need to convert the scene into a transparency and then illuminate that transparency with a collimated normally incident laser beam is unnecessarily complicated if one wants to record the Fourier transform of a passively illuminated incoherent scene in real time. Breckinridge ${ }^{2}$ in 1972 conceived of the idea for a white-light interferometer to enable the recording the twodimensional spatial frequency Fourier transform of a passively illuminated white light scene over a broad wavelength band. He called the instrument a "Coherence Interferometer". In this paper we describe the theory and the development of the hardware and its applications to optical image processing, applications to atmospheric physics, and applications to the recording of the first two-dimensional images of the Michelson stellar interferometer fringes and also applications to imaging at the diffraction limit with large ground based telescopes through atmospheric turbulence.

\section{PRINCIPLES OF THE COHERENCE INTERFEROMETER}

\subsection{The hardware}

A qualitative description of the operation of the interferometer is given below, followed by a theoretical analysis of its operation. The interferometer manipulates the complex (amplitude and phase) wavefront as shown in Fig 1 below.

Tribute to Joseph W. Goodman, edited by H. John Caulfield, Henri H. Arsenault, Proc. of SPIE Vol. 8122, $812206 \cdot$ ㅇ 2011 SPIE · CCC code: 0277-786X/11/\$18 · doi: 10.1117/12.890537 


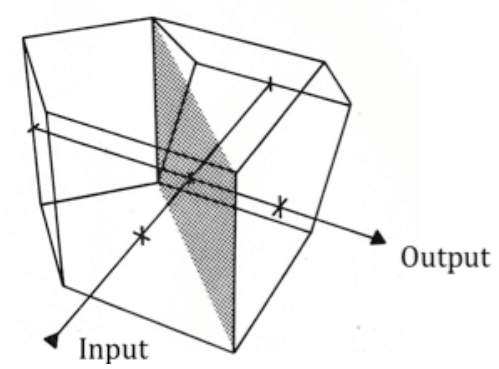

Figure 1 shows the interferometer cube prism coherence interferometer.

In Fig. 1 we see the input wavefront is from the lower left and the output wavefront is to the right. This prism appears similar to the Michelson interferometer cube, but the flat surfaces are replaced with crossed roof prisms. A wavefront enters from the lower left, reflects from the beamsplitter and passes into the left arm of the interferometer. This wavefront strikes a roof and the electromagnetic field is rotated by 180 degrees about the vertical axis. That portion of the wavefront that passes straight through the beamsplitter, strikes another roof and this electromagnetic field is rotated by 180 degrees about the horizontal axis. Both wavefronts then pass back to the beamsplitter and out of the prism assembly. It is important that the optical path lengths in each arm of the interferometer be equal to obtain system performance in white light. This equality is achieved by shearing one prism across the beamsplitter face until the white light fringe is obtained while viewing through a spectrometer such as a hand spectroscope and changing the OPD until channel spectra open up to the bright fringe filling the spectrometer field.

The interferometer cube prism assembly is placed within an optical system as shown in Fig 2 below.

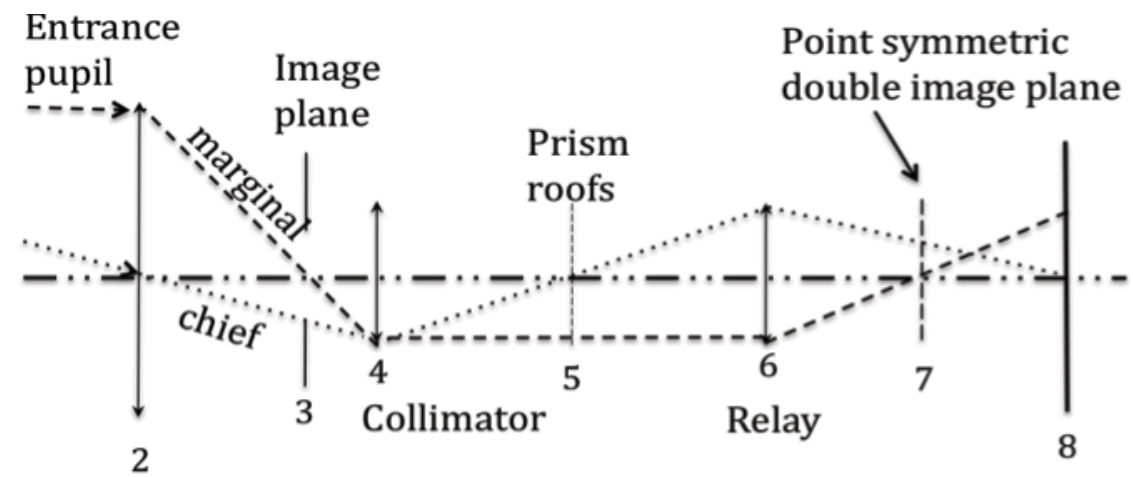

\section{Pupil image with fringes}

Fig 2 shows a diagram of an optical imaging system with the coherence interferometer located at an image of the entrance pupil.

The marginal and chief rays are shown traced through the optical system.

In Fig 2 light enters the system from the left at the entrance pupil (plane 2). An image of the object space is formed at the image plane (plane 3). After the image plane a field lens is located to collimate light from the image plane and to reimage the entrance pupil onto plane 5. At plane 5 we position the Coherence Interferometer and adjust its position so that that an image of the entrance pupil falls onto the prism roofs. Two images of the entrance pupil are formed, one on each of the two orthogonal roofs. The complex wavefronts are folded and flipped at plane 5 and pass onto relay optics at plane 6. Midway between planes 6 and 7 two images of the image plane appear, one rotated 180 degrees with respect to the other. At plane 7 white light fringes appear across an image of the pupil plane provided there is path length equality in the prisms.

Figure 3 shows an isometric view of the system given in Fig. 2 to provide an additional perspective on how the interferometer system operates. 


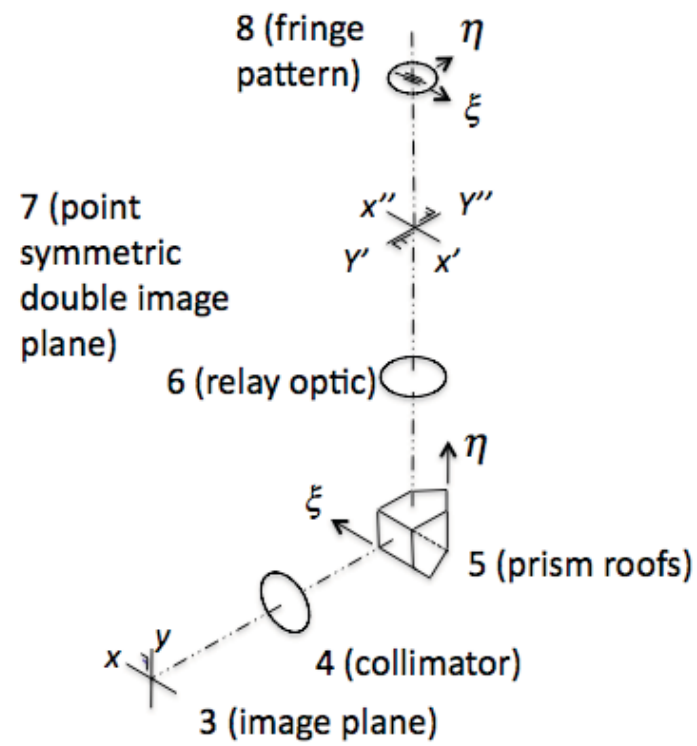

Fig. 3 shows an isometric drawing of the layout of the coherence interferometer, positioned in a telescope imaging system. The numbers in this figure correspond to the numbers used in Fig. 2.

In Fig 3. plane three is the telescope (fore-optics) image plane and it shows the image plane coordinates and the letter $\mathrm{F}$ in the upper right quadrant of the image plane when viewed from the right looking to the left, into the direction of the light travel. The collimator at plane 4 relays an image of the system pupil into the prism roofs of the coherence interferometer shown at plane 5. An optic at plane 6 relays the image plane (plane 3) to plane seven. The coherence interferometer prism assembly has created two images of the letter $\mathrm{F}$ at plane 7 . These two images have point symmetry about the axis of the system defined by the apparent intersection point of the two roof prisms. If the optical path lengths of the two arms of the coherence interferometer are equal, then each point on the letter F in the "prime" coordinate system is coherent with the same point in the "double-prime" coordinate system. If we examine a single elemental point on the letter $\mathrm{F}$ in plane 3 we see that it is mapped into two points at plane 7 . Because the coherence interferometer is adjusted for white light each of these points is coherent with the other and a fringe pattern is formed at plane 8 . The amplitude of the fringe pattern is proportional to the intensity of light at each elemental point in the letter $\mathrm{F}$ at plane 3.

The envelop of the fringe intensity distribution across the image plane 7 is then the Fourier transform of the spatial frequency structure in object space. The image at plane 3 is confined to the upper right hand quadrant. Note that since the amplitude image at the "doubled image plane" is point symmetric, the coefficients on the sine (odd terms) transform are zero and the intensity distribution at image plane 8 is the cosine (even terms) transform.

\subsection{Theory of the FTS}

The theory for the less familiar coherence interferometer can be understood by referencing the more familiar Fourier transform spectrometer (FTS) used to record interferogram information which is then converted to power spectra for physical analysis of electron transitions in atoms and vibration-rotation structure in molecules by spectroscopists.

Fig. 4 shows a view of a Twyman-Green or a Fourier Transform Spectrometer (FTS) ${ }^{3}$. This configuration was developed for optical testing by Twyman and Green ${ }^{4}$ and, independently has found application in Fourier transform spectrometers for spectral analysis of radiation in the visible and infrared ${ }^{5}, 6$. The rectangle just after lens 1 represents a volume beam of light in time entering the interferometer. The complex wavefront of this beam is divided at the beamsplitter. Part reflects from the beam splitter and travels to mirror $\mathrm{M}_{2}$, is reflected and passes back through the beamsplitter. The other part is transmitted through the dielectric beamsplitter and its substrate, reflects from mirror 
$\mathrm{M}_{1}$, and returns to pass through the beamsplitter substrate and reflect from the dielectric beamsplitter. The "cylinder" of light entering the interferometer is divided into two parts, one phase delayed relative to the other.

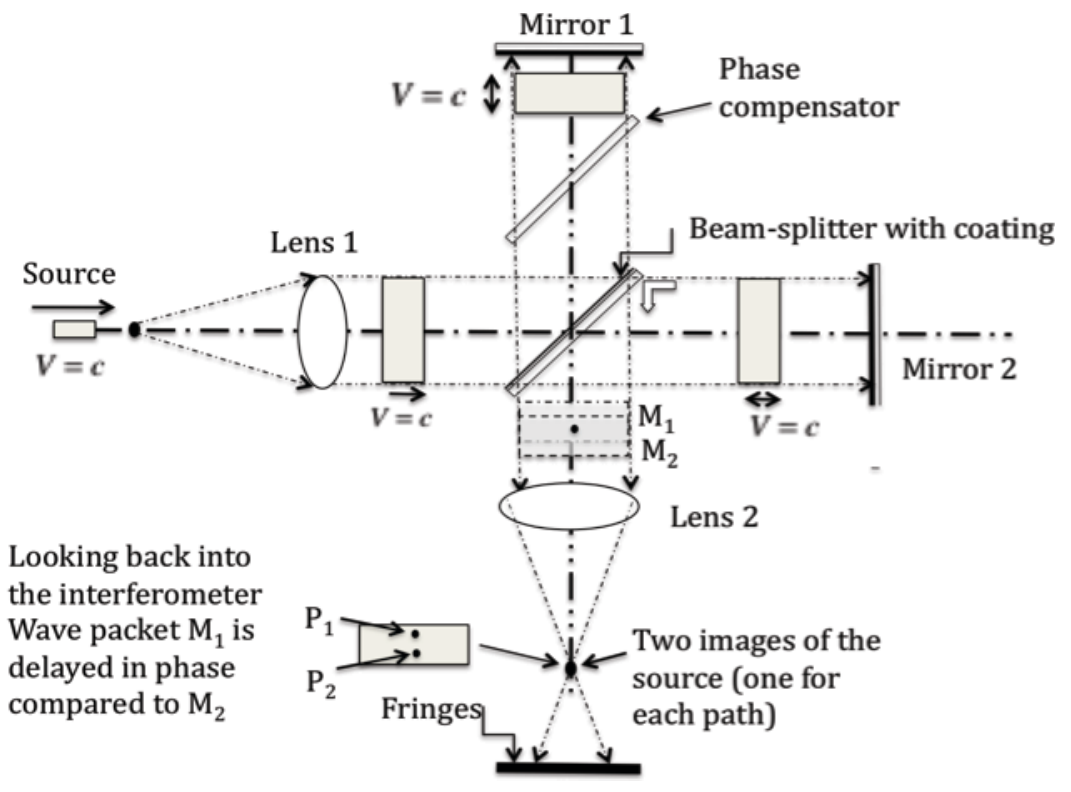

Fig 4 The Fourier transform spectrometer sometimes referred to as the Twyman-Green interferometer.

In Fig. 4, the solid cylinder has passed through the interferometer arm $\mathrm{M}_{2}$ and the dotted cylinder has passed through the interferometer arm $M_{1}$ of the interferometer. $P_{1}$ is shows retarded or shifted in phase to lag behind the wavefront at $\mathrm{P}_{2}$. The wavefront at the detector is the sum of the complex wavefront at point $\mathrm{P}_{1}$ plus that at point $\mathrm{P}_{2}$. The detection process records the intensity, which is the sum of the complex wavefronts $\mathrm{P}_{1}$ and $\mathrm{P}_{2}$ modulus squared.

We represent light entering the interferometer by the analytic signal $V(\xi, \eta ; t)$, where $\xi, \eta$ are orthogonal space coordinates perpendicular to the direction of propagation and $t$ is the time. Fig. 4 shows that the path from the beamsplitter to mirror $\mathrm{M}_{2}$ is longer than the path from the beamsplitter to mirror $\mathrm{M}_{1}$. Let $V_{1}\left(\xi, \eta ; t_{1}\right)$ be the analytic signal for the complex wavefront reflecting from mirror $\mathrm{M}_{1}$, and $V_{2}\left(\xi, \eta ; t_{2}\right)$ be the analytic signal for the complex wavefront from mirror $\mathrm{M}_{2}$. Then the intensity as a function of position $\xi, \eta$ measured by the detector averaged over time intervals long compared with the temporal period of the light is

$$
I\left(\xi, \eta ; t_{1}, t_{2}\right)=\left\langle\left|V_{1}\left(\xi, \eta ; t_{1}\right)+V_{2}\left(\xi, \eta ; t_{2}\right)\right|^{2}\right\rangle
$$

And expanding the brackets we obtain:

$$
\begin{aligned}
& I\left(\xi, \eta ; t_{1}, t_{2}\right)=\left\langle\left|V_{1}\left(\xi, \eta ; t_{1}\right)\right|^{2}\right\rangle+\left\langle\left|V_{2}\left(\xi, \eta ; t_{2}\right)\right|^{2}\right\rangle \\
& +\left\langle V_{1}\left(\xi, \eta ; t_{1}\right) \cdot V_{2}^{*}\left(\xi, \eta ; t_{2}\right)\right\rangle+\left\langle V_{2}\left(\xi, \eta ; t_{2}\right) \cdot V_{1}^{*}\left(\xi, \eta ; t_{1}\right)\right\rangle
\end{aligned}
$$

The brackets indicate time averages long compared to the frequency of oscillation of the optical electromagnetic wave, $\boldsymbol{*}$ means complex conjugate, and || means the modulus.

The statistics that represent the radiation are stationary in time and we can write the expressions in Eq. (2) as

$$
V_{1}\left(\xi, \eta ; t_{1}\right) \cdot V_{2}^{*}\left(\xi, \eta ; t_{2}\right)=V_{1}(\xi, \eta ; t) \cdot V_{2}^{*}(\xi, \eta ; t+\tau)
$$


And

$$
V_{2}\left(\xi, \eta ; t_{2}\right) \cdot V_{1}^{*}\left(\xi, \eta ; t_{1}\right)=V_{2}(\xi, \eta ; t) \cdot V_{1}^{*}(\xi, \eta ; t+\tau)
$$

Where we let $\tau=t_{1}-t_{2}$.

We use the notation ${ }^{7}$ :

$$
\begin{gathered}
\Gamma(\tau)=\left\langle V_{1}(\xi, \eta ; t) \cdot V_{2}^{*}(\xi, \eta ; t+\tau)\right\rangle \\
\text { And } \\
\Gamma^{*}(\tau)=\left\langle V_{2}(\xi, \eta ; t) \cdot V_{1}^{*}(\xi, \eta ; t+\tau)\right\rangle
\end{gathered}
$$

Where $\Gamma(\tau)$ is the temporal mutual coherence function with zero spatial shear and with time delay $\tau$.

$\Gamma(\tau)$ represents the cross correlation of the two fields, at the same spatial point $\xi, \eta$, but with time delay $\tau$. In equation 2 , the first two terms represent a constant background level. The signal is then a constant background level with modulation $\Gamma(\tau)+\Gamma^{*}(\tau)$.

In this case of the Fourier transform spectrometer (interferometer) the radiation from each arm of the interferometer is combined and detected, that is we take the modulus squared of the sums of the complex amplitudes to obtain

$$
I(\xi, \eta ; \tau)=\frac{1}{2}[I(\xi, \eta)]+\frac{1}{4}\left[\Gamma(\tau)+\Gamma^{*}(\tau)\right]
$$

We now examine the sum given by $\Gamma(\tau)+\Gamma^{*}(\tau)$ in Eqs $5 \& 6$.

The temporal frequency mutual coherence function is defined ${ }^{8}$ as

$$
\begin{aligned}
\Gamma(\tau) & =\int_{0}^{\infty} \hat{\Gamma}(v) \exp [-2 \pi i v \tau] d v \\
\Gamma^{*}(\tau) & =\int_{0}^{\infty} \hat{\Gamma}^{*}(v) \exp [+2 \pi i v \tau] d v
\end{aligned}
$$

Where $\hat{\Gamma}(v)$ is the power spectrum of the radiation. Using Eqs. 8 and 9, the last two terms in Eq. 7 become

$$
\begin{aligned}
& \Gamma(\tau)+\Gamma^{*}(\tau)= \\
& \int_{0}^{\infty} \hat{\Gamma}(v) \exp [-2 \pi i v \tau] d v+\int_{0}^{\infty} \hat{\Gamma}^{*}(v) \exp [+2 \pi i v \tau] d v .
\end{aligned}
$$

We note that the power spectrum is real, then

$$
\Gamma(\tau)+\Gamma^{*}(\tau)=\int_{0}^{\infty} 2 \operatorname{Re}[\Gamma(v)] \cos (2 \pi v \tau) d v
$$

And we see that the modulation found from Eq. 7 is the cosine Fourier transform of the power spectrum. Note this is true only if the interferometer is perfectly phase compensated. That is, the optical-path-distances for all wavelengths are the same in each arm of the interferometer that is $v \neq f(\tau)$. 
We also make the assumption that the source is quasimonochromatic, that is appreciably different from zero only for the spectral components $v$, which satisfy the inequality $|v-\bar{v}|<\Delta v$ where $\Delta v$ is a spectral bandpass that satisfies

$\frac{\Delta v}{\bar{v}} \ll 1$, and $\bar{v}$ is the mean frequency.

Then we find,

$$
\Gamma(\tau)+\Gamma^{*}(\tau)=2\left|\Gamma_{o}\left(\tau_{o}\right)\right| \cdot \cos \left(2 \pi \bar{v} \tau+\Phi_{\Gamma}(\tau)\right)
$$

And we see that the fringe envelope on the cosine term reveals information on the real part of the temporal frequency mutual coherence function.

Returning to equation 7, we then see the intensity measured at the output of the interferometer as a function of $\tau$ or path difference is:

$$
I(\xi, \eta ; \tau)=\frac{1}{2}[I(\xi, \eta)]+\frac{1}{4}\left[\Gamma(\tau)+\Gamma^{*}(\tau)\right]
$$

And

$$
\begin{aligned}
& I(\xi, \eta ; \tau)= \\
& \frac{1}{2}[I(\xi, \eta)]+\frac{1}{2}\left|\Gamma_{o}(\tau)\right| \cos \left(2 \pi \bar{\nu}+\Phi_{\Gamma}(\tau)\right)
\end{aligned} .
$$

The Fourier transform of the term $\left|\Gamma_{o}(\tau)\right|$ is the spectrum of the source. This is identical within a scaling factor to the spectrum obtained using a diffraction grating spectrometer.

\subsection{Theory of the Coherence Interferometer}

A comprehensive analysis of the image forming properties of the coherence interferometer (180-degree rotational shear interferometer) appears in the PhD dissertation from the College of Optical Sciences ${ }^{9}$. Consider Fig. 1, which shows the construction of the Coherence Interferometer and Fig 2, which shows how the interferometer is used in an optical system. Examine Fig. 1 light enters the prism from the left and is wavefront divided with part transmitting into the roof positioned to the right. The transmitted wavefront is reflected top to bottom, and returns to the beamsplitter to reflect out of the system towards the viewer. Consider the wavefront that enters the system and reflects from the beam splitter to pass into the roof prism in the upper left of fig. 1. This wavefront is flipped left to right and reflects back to pass through the beamsplitter parallel to the wavefront that passed into the upper right arm and was flipped top to bottom

We represent the light entering by the analytic signal used earlier for the FTS: $V(\xi, \eta ; t)$, where $\xi, \eta$ are space coordinates and $t$ is time. The analytic signal $V_{1}(-\xi,+\eta ; t)$ represents the wavefront that is flipped about the $\xi$ axis shown in Fig. 3. The analytic signal $V_{2}(+\xi,-\eta ; t)$ represents the wavefront that is flipped about the $\eta$ axis in Fig. 3. The out put intensity as measured by the detector at the output is

$$
\begin{gathered}
I(\xi, \eta)=\left\langle\left|V_{1}(-\xi,+\eta ; t)+V_{2}(+\xi,-\eta ; t)\right|^{2}\right\rangle \\
I(\xi, \eta)=\left\langle\left|V_{1}(-\xi,+\eta ; t)\right|^{2}\right\rangle+\left\langle\left|V_{2}(+\xi,-\eta ; t)\right|^{2}\right\rangle+ \\
\left\langle V_{1}(-\xi,+\eta ; t) \cdot V_{2}^{*}(+\xi,-\eta ; t)+V_{2}(+\xi,-\eta ; t) \cdot V_{1}^{*}(-\xi,+\eta ; t)\right\rangle
\end{gathered}
$$

Note that we adjust the interferometer to be white-light compensated, and all the times are the same and hence we will drop the $t$ dependence. We use the assumption that the system operates in quasimonochromatic light. The interferometer is aligned for the "white light" fringe and therefore, 


$$
t=0
$$

Following the definition of mutual coherence function ${ }^{10}$, from Eq. 3 we write:

$$
\begin{aligned}
& \Gamma(2 \xi, 2 \eta)=\left\langle V_{1}(-\xi,+\eta) \cdot V_{2}^{*}(+\xi,-\eta)\right\rangle \\
& \Gamma^{*}(2 \xi, 2 \eta)=\left\langle V_{2}(+\xi,-\eta) \cdot V_{1}^{*}(-\xi,+\eta)\right\rangle
\end{aligned}
$$

where $\Gamma(2 \xi, 2 \eta)$ is the spatial frequency mutual coherence function for zero time delay. The argument in the spatial mutual coherence function is the point separation, or shear distance. If the beamsplitter has complex amplitude and phase reflectivity $R$ and complex amplitude and phase transmissivity $T$, then

$$
\left.\begin{array}{l}
V_{1}(-\xi,+\eta)=T \cdot V_{1}(-\xi,+\eta) \cdot R \\
V_{2}(+\xi,-\eta)=R \cdot V_{2}(+\xi,-\eta) \cdot T
\end{array}\right\}
$$

The interferometer shown in Fig. 10.5 yields a shear of $2 \xi, 2 \eta$ for each point $\xi, \eta$. If T and R are optimized, then $R=T=1 / \sqrt{2}$, and the intensity from Eq. 15 becomes

$$
I(\xi, \eta)=\frac{1}{2}\left[\langle I\rangle_{\xi, \eta}\right]+\frac{1}{4}\left[\Gamma(2 \xi, 2 \eta)+\Gamma^{*}(2 \xi, 2 \eta)\right],
$$

where $<I>_{\xi, \eta}$ is intensity integrated over the pupil and represents a spatial frequency DC offset. This term has no spatial structure across the pupil. The second part of eq. 19 involves only the spatial dependence of the mutual coherence function and is similar in form to Eq. 7 derived for the temporal frequency interferometer: Fourier transform spectrometer.

Examine the sum: $\Gamma(2 \xi, 2 \eta)+\Gamma^{*}(2 \xi, 2 \eta)$ in Eq. 19. By definition of the spatial mutual coherence function we can write:

$$
\left.\begin{array}{l}
\Gamma(\xi, \eta)=\int_{-\infty}^{\infty} \int_{-\infty}^{\infty} \hat{\Gamma}(x, y)^{-2 \pi i(x \xi+y \eta)} d x d y \\
\Gamma^{*}(\xi, \eta)=\int_{-\infty}^{\infty} \int_{-\infty}^{\infty} \hat{\Gamma}^{*}(x, y)^{-2 \pi i(x \xi+y \eta)} d x d y
\end{array}\right\} .
$$

$\hat{\Gamma}(x, y)$ is real and $\hat{\Gamma}(x, y)=\hat{\Gamma}^{*}(x, y)$. Therefore, $\Gamma(2 \xi, 2 \eta)+\Gamma^{*}(2 \xi, 2 \eta)=\int_{-\infty}^{\infty} \int_{-\infty}^{\infty} 2|\hat{\Gamma}(x, y)| \cos (4 \pi \xi x+4 \pi \xi y) d x d y$, and we see that the envelop of the cosine in the space variable $\xi, \eta$ is the cosine Fourier transform of the function $\hat{\Gamma}(x, y)$.

We now define a new concept. We assume that the source is quasimonoplanatic, that is, that $\hat{\Gamma}(x, y)$ is appreciably different from zero only for those components of $x, y$ which satisfy the inequalities $|x-\bar{x}|<<\bar{x},|y-\bar{y}|<<\bar{y}$ and $\frac{\Delta x}{\bar{x}}<<1, \frac{\Delta y}{\bar{y}}<<1$, where $\bar{x}$ and $\bar{y}$ is the source centroid. Then we can remove the term from the integral above and write:

$$
\Gamma(2 \xi, 2 \eta)+\Gamma^{*}(2 \xi, 2 \eta)=2\left|\Gamma_{0}(2 \xi, 2 \eta)\right| \cdot \cos \left[4 \pi(\xi, x+\eta y)+\phi_{\Gamma}(2 \xi, 2 \eta)\right] \cdot
$$

We are then able to write Eq. 19 as:

$$
I(\xi, \eta)=\frac{1}{2}\left[<I>_{\xi, \eta}\right]+\frac{1}{2}\left|\Gamma_{0}(2 \xi, 2 \eta)\right| \cos \left[4 \pi(\xi x+\eta y)+\phi_{\Gamma}(2 \xi, 2 \eta)\right] .
$$


In Eq. (22) the term $\left|\Gamma_{0}(2 \xi, 2 \eta)\right|$ is seen to be the spatial spectrum of the source, were it to be centered on the axis. That is, to first order tilt errors have been removed.

\section{APPLICATIONS}

Following the development of the theory by the author he designed and built and a coherence interferometer and applied this new instrument to several scientific and image processing applications. These are described here.

\subsection{Construction of the Interferometer -}

Fig 5 below shows a detailed image of the interferometer.

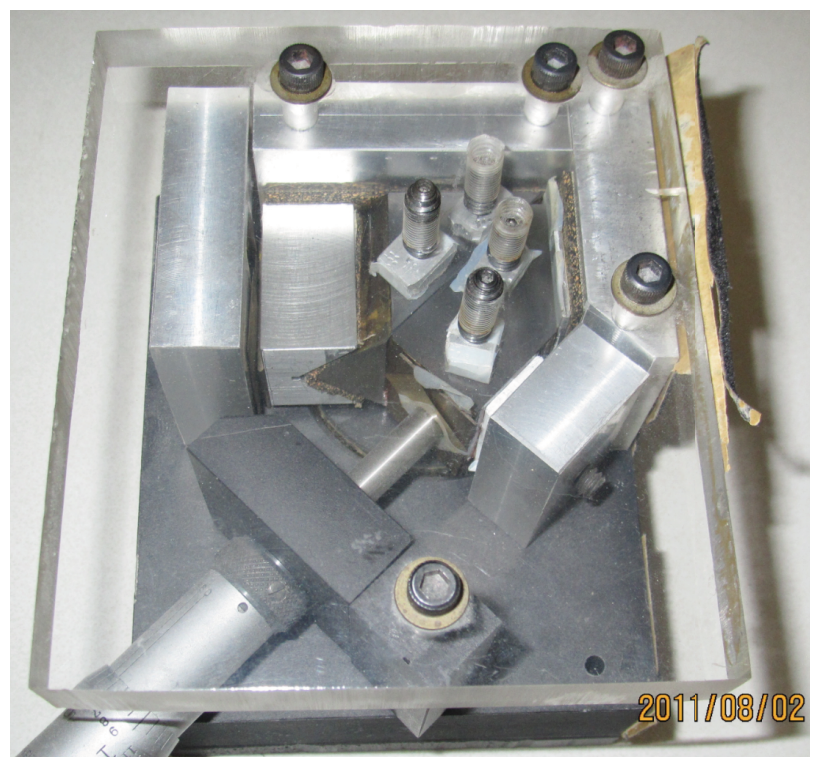

Fig. 5 shows a view from the top of the coherence interferometer. In this view light enters downward from the top into the interferometer prism assembly and exits to the right. The top of the interferometer casing is a thick plate of Lucite. Within the assembly, the prism with its roof horizontal is to the left and the prism with its roof vertical is to the right. The horizontal prism to the left has its roof cradled in a cork pad to avoid direct contact between glass and aluminum. The compound micrometer screw shown on the lower left pushes prism with it's roof vertical to the upper right against spring loaded screws (not shown) to change the OPD of one arm relative to the other within the interferometer to achieve white light performance. The four spring-loaded screws press each prism down onto a $1 / 10$-wave flat reference flat reference surface. Low viscosity silicon oil is at each glass-to-glass surface.

\subsection{The Michelson Stellar Interferometer fringes -}

The McMath-Pierce solar telescope at Kitt Peak National Observatory was used in 1974 to record the first direct images of the Michelson Stellar Interferometer fringes ${ }^{11}$. An instrument was built incorporating the coherence interferometer and the relay optics shown in Fig. 3. A video recording was desired to make a quantitative measure of atmospheric turbulence. This was before the development of the CCD camera. Scanning image converter tubes such as vidicons and plumbicons were not sensitive enough to record the moving fringes from even the brightest star. A 16 $\mathrm{mm}$ movie camera with enhanced Tri-X (ASA $=>1,000)$ movie film framed at a 20 milliseconds frame rate was sensitive enough record the fringes moving back and forth across the pupil of the telescope when viewing a $1^{\text {st }}$ magnitude star and compressing the image on the film to a few $\mathrm{mm}$. The Michelson stellar interferometer fringes from the bright star Sirius, $\alpha$ Canis Majoris (the brightest star in the sky, at $\mathrm{M}_{\mathrm{v}}=-1.46$ ) were observed visually several times without being able to record fringes. The latitude of Kitt Peak was too far north to allow good enough seeing to record the fringes from this low declination star. The 0 magnitude star $\alpha$ Lyr (Vega) was far enough north and fringes were recorded on tri-X movie film in the summer of 1973 during a night of excellent seeing. Figure 4 shows one frame of the video sequence for $\mathrm{Vega}^{12}$. Fringes are seen passing across the pupil at about $30^{\circ}$ from the horizontal from the upper left to the lower right. The crossed roofs of the prisms are clearly seen in fig 4. 


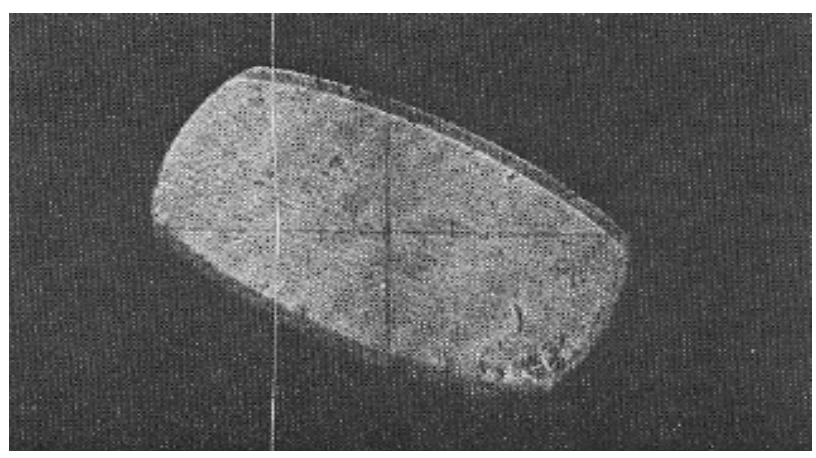

Fig. 6 shows the pupil of the McMath-Pierce solar telescope at Kitt peak national observatory with Michelson stellar interferometer fringes running left to right draped across the pupil. The fringes from Vega or $\alpha$ Lyr were changing with time and recorded at the image of the pupil at plane 11 in Fig 3. This solar telescope uses a heliostat configuration and is designed to look at the sun within the ecliptic. The pupil of the telescope appears flattened because of vignetting when pointing the heliostat as far to the north as the declination of Vega.

\subsection{Atmospheric physics -}

The instrument was used to make three measurements of the properties of the Earth's atmosphere. Fig 5 shows measurements (individual points) of the fringe amplitude in waves as a function point separation in meters ${ }^{13}$. The solid line shows the theoretical dependence provided by the phase structure function for the atmosphere derived theoretically by Fried ${ }^{14}$. This was the first time direct measurement of phase perturbations in the atmosphere were made.

These measurements were then used to calculate the order of interference in astronomical patterns. Astronomical speckle patterns from bright stars were recorded using the Kitt Peak Speckle camera ${ }^{15}$ at the 4 -meter Mayall telescope and were used to determine experimentally ${ }^{16}$ that astronomical speckle patterns contain speckles formed from interference orders 1, 2 and 3. Astronomers had been incorrectly assuming that speckles were multiple "white-light" images of the nearly unresolved star and creating images of stellar surfaces by co-adding speckles ${ }^{17}$. Theory was also developed $^{18,19}$ to show that the coherence interferometer enables imaging through atmospheric turbulence at the diffraction limit of large telescopes.

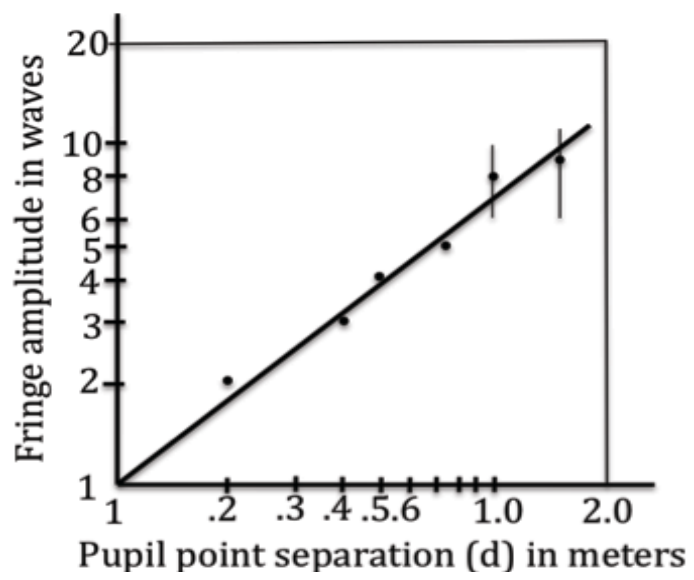

Fig 7 shows a plot (the points) of fringe amplitude in waves $(\lambda)$ as a function of pupil point separation (d) in meters. The solid line is the theoretical prediction.

C. and F. Roddier ${ }^{20}$ continued this work in astronomy and atmospheric physics by using a rotational shear interferometer of their own design.

\subsection{Optical image processing -}


We showed in the theory above that the coherence interferometer enables the spatial frequency Fourier transform of passively illuminated white light scenes. E. Ribak collaborated with J. Breckinridge in his laboratory at JPL to demonstrate experimentally that spatial frequency Fourier transforms of white light scenes can be recorded and determined the signal to noise ratio rules ${ }^{21}$. Breckinridge gave a comparison between different optical processing architectures including the coherence interferometer in the review ${ }^{22}$.

\subsection{Exoplanets -}

Recently the achromatic rotation-shearing coronagraph was developed to provide a variable inner working angle that is easily tunable to ensure the observation of Solar-like systems with extremely large telescopes ${ }^{23}$. The coherence interferometer is used as a fringe nulling interferometer to null the central planet and make direct observations of exoplanets by Serabyn, et. al. ${ }^{24}$

\section{SUMMARY AND ACKNOWLEDGEMENTS}

A white-light compensated rotational shear interferometer was developed with 90-degrees of shear. Theory was developed for the device and it was tested and applied to problems in optical information processing, astrophysics and atmospheric science. Since 1976 there have been over 200 scientific papers written using a coherence interferometer or rotational shear interferometer to acquire the data.

The author would like to thank Professor Sergio Pellegrino at CALECH and Dean Jim Wyant of the College of Optical Sciences for their support during the preparation of this manuscript. Deep appreciation goes to Professors Joseph Goodman and Jack Gaskill for their skill in making a complex subject clear and concise.

\footnotetext{
1 Goodman, J. W. , [Fourier Optics] McGraw Hill (1968).

2 Breckinridge, J. B. "Coherence Interferometer and astronomical applications", Applied Optics 11, 2996-2998, (1972).

3 Griffiths, P. and J. D. Haseth, [Fourier Transform Infrared Spectrometry]. New York, Wiley (2007).

4 Twyman, F. and A. Green [Interferometer] U. P. Office. United Kingdom. 103832, (1916).

5 Saha, S. "Modern optical astronomy: technology and impact of interferometry" Reviews of Modern Physics 74: 551-559, (2002).

6 Breckinridge, J. and R. A. Schindler "First-Order Optical Design for Fourier Spectrometers" [Spectrometric Techniques]. G. A. Vanasse. New York, Academic Press. 2: 63-125, (1981).

7 Mandel, L. and E. Wolfe "Coherence Theory” Reviews of Modern Physics 37: 231-287, (1965).

8. Beran, M. J. and G. B. Parrent [Theory of Partial Coherence]. Englewood Cliffs, NJ, Prentice-Hall, (1964)

9 Breckinridge, James B. [The Spatial Structure Analyzer and its Astronomical Applications], 212 page dissertation for the PhD in Optical Sciences, University of Arizona, Tucson, AZ (1976)

10 Born, M. and E. Wolf [Principals of Optics] pp 564. 7th edition, (2005)

11 Breckinridge, J. B. "Two-dimensional white-light coherence interferometer", Applied Optics, 13, 2760-2762, (1974)

12 Breckinridge, J. B. "Two Dimensional White Light Coherence Interferometer." Applied Optics 13: 2760-2762, (1974).

13 Breckinridge, J. B. "Measurement of amplitude of phase excursions in the Earth's atmosphere ” JOSA 66, 143-144, (1976)

14 Fried, D. L., Statistics of a geometric representation of wavefront distortion, JOSA 55 1427-1432, (1965)

15 J. B. Breckinridge H. A. McAlister and W. G. Robinson "The Kitt Peak Speckle Camera." Applied Optics, 18 1034-1041, (1979).

16 Breckinridge, J. B. "Interference in astronomical speckle patterns", JOSA 66, 1240-1242, (1976),

17 Worden, S. P., J. W. Harvey and C. R. Lynds "Reconstructed images of Alpha Orionis using stellar speckle interferometry,, JOSA 66, 181, (1976)

18 Breckinridge, J. B, "Obtaining information through the atmosphere at the diffraction limit of a large aperture" JOSA 65, 755-759, .(1976)

19 Breckinridge, J. B. and J. Burke "Passive imaging through turbulent atmosphere - fundamental limits on spatial frequency resolution of a rotational shearing interferometer" JOSA $68,67-77,(1978)$

20 Roddier, C., F. Roddier, "Diffraction limited imaging of unknown objects through fixed unknown aberrations using interferometry" JOSA-A Optics Image Science and Vision, 7 , issue 10 1824-1833. (1990)

21 Ribak, E. C. Roddier, F. Roddier, J. B. Breckinridge "Signal-to-noise limitations in white light holography", Applied Optics 27 1183-1186, (1988)

22 Breckinridge, J. B. "Review of image correlation systems - hybrid and optical; Critical Reviews" Conference on applications of electronic imaging. SPIE Vol. 1082 pages 116124. (1990)

23 Aime, C., G. Ricort, A. Carlotti, Y. Rabbia, and J. Gay “ARC: an Achromatic Rotation-shearing Coronagraph,, Ast. \& Astroph. 517, A55, DOI 10.1051/0004-6361/200912939, (2010)

24 Serabyn, E., D. Mawet and R. Burruss, “An image of an exoplanet separated by two diffraction beamwidths from a star” Nature 464, 1018-1020, DOI: 10.1038
} 